\title{
Conditions for Singular Incidence Matrices
}

\author{
WILLEM H. HAEMERS \\ Department of Econometrics and O.R., Tilburg University, Tilburg, The Netherlands
}

haemers@uvt.nl

Received July 28, 2003; Revised March 30, 2004; Accepted March 30, 2004

\begin{abstract}
Suppose one looks for a square integral matrix $N$, for which $N N^{\top}$ has a prescribed form. Then the Hasse-Minkowski invariants and the determinant of $N N^{\top}$ lead to necessary conditions for existence. The BruckRyser-Chowla theorem gives a famous example of such conditions in case $N$ is the incidence matrix of a square block design. This approach fails when $N$ is singular. In this paper it is shown that in some cases conditions can still be obtained if the kernels of $N$ and $N^{\top}$ are known, or known to be rationally equivalent. This leads for example to non-existence conditions for self-dual generalised polygons, semi-regular square divisible designs and distance-regular graphs.
\end{abstract}

Keywords: incidence matrix, Bruck-Ryser-Chowla theorem, generalised polygon, divisible design, distanceregular graph

\section{Introduction}

Consider a square 2- $(v, k, \lambda)$ design with incidence matrix $N$. (We prefer the name 'square' to 'symmetric', since $N$ is not necessarily symmetric.) Then $N N^{\top}=\lambda J_{v}+(k-\lambda) I_{v}$, where $J_{v}$ is the $v \times v$ all-ones matrix and $I_{v}$ is the identity matrix of size $v$. The BruckRyser-Chowla theorem is based on two observations (see for example [7] p. 223). The first one is that $\operatorname{det} N=\operatorname{det} N^{\top}$ is an integer. Therefore $\operatorname{det}\left(\lambda J_{v}+(k-\lambda) I_{v}\right)$ is an integral square, hence $k-\lambda$ is a square if $v$ is even. The other observation is that, since $N$ is a non-singular rational matrix, $\lambda J_{v}+(k-\lambda) I_{v}$ is rationally congruent to $I_{v}$, and therefore these two matrices have the same Hasse-Minkowski invariants. These invariants can be expressed in terms of $v, k$ and $\lambda$ from which it follows that for odd $v$ the Diophantine equation $(k-\lambda) X^{2}+(-1)^{(v-1) / 2} \lambda Y^{2}=Z^{2}$ has an integral solution different from $X=Y=Z=0$. Similar approaches work for other square incidence structures for which the determinant or the Hasse-Minkowski invariants of $N N^{\top}$ are known. See for example [7], Chapter 12. It is clear that this approach gives no conditions if $N$ is singular. In the present paper we modify the mentioned approach such that we still find conditions for singular $N$. The key lemma is a simple trick that changes a singular $N$ into a non-singular matrix $M$ in such a way that for some types of designs it is still possible to compute the Hasse-Minkowski invariants or the (square free part of the) determinant of $M M^{\top}$.

Lemma 1 Suppose $N$ is a real $v \times v$ matrix of rank $v-m$. Let $Z$ be a real $v \times v$ matrix of rank $m$, such that $N^{\top} Z=N Z^{\top}=O$. Define $M=N+Z$, then

(i) $M M^{\top}=N N^{\top}+Z Z^{\top}$, 
(ii) the eigenvalues of $M M^{\top}$ are the positive eigenvalues of $N N^{\top}$ together with the positive eigenvalues of $Z Z^{\top}$,

(iii) $M M^{\top}$ is non-singular.

Proof: Part (i) is staightforward. To prove (ii), first notice that $N N^{\top}$ and $Z Z^{\top}$ commute, so they have a common orthogonal basis of eigenvectors. Suppose $\mathbf{v}$ is such an eigenvector that corresponds to a positive eigenvalue of $N N^{\top}$. Then $\mathbf{v}$ is orthogonal to the kernel of $N N^{\top}$, which is the span of the columns of $Z$. Hence $Z^{\top} \mathbf{v}=\mathbf{0}$, so the corresponding eigenvalue of $Z Z^{\top}$ equals 0 . Similarly, a positive eigenvalue of $Z Z^{\top}$ corresponds to an eigenvalue 0 of $N N^{\top}$. This proves (ii), since $N N^{\top}$ has $v-m$ positive eigenvalues, and $Z Z^{\top}$ has $m$ positive eigenvalues. Statement iii follows because $M M^{\top}$ has only positive eigenvalues.

For a given $N$, a matrix $Z$ with the required properties always exists. One way to make such a $Z$ is the following. Take rational $v \times m$ matrices $L$ and $R$, whose columns form a basis for the left and the right kernel of $N$, respectively. Then $\operatorname{rank} L=\operatorname{rank} R=m$ and $N^{\top} L=N R=O$. Therefore $Z=L R^{\top}$ has the desired properties.

In the coming sections we will consider two kinds of square designs for which something new can be said: Self-dual designs and semi-regular square divisible designs.

\section{Self-dual designs}

Consider two $m$-dimensional subspaces $V$ and $W$ of the vectorspace $\mathbb{Q}^{v}$. Let $L$ and $R$ be rational $v \times m$ matrices whose columns span $V$ and $W$, respectively. We call the subspaces $V$ and $W$ rationally equivalent if $L^{\top} L$ and $R^{\top} R$ are rationally congruent matrices, which means that $S^{\top} L^{\top} L S=R^{\top} R$ for some non-singular rational matrix $S$. Note that rational equivalence of vectorspaces does not depend on the choice of $L$ and $R$.

Lemma 2 Let $N$ be a rational $v \times v$ matrix. If the left kernel and the right kernel of $N$ are rationally equivalent then the product of the non-zero eigenvalues of $N N^{\top}$ is a rational square.

Proof: Let $L$ and $R$ be rational $v \times m$ matrices whose columns form a basis for the left and the right kernel of $N$, respectively. Put $Z=L R^{\top}$. Then $Z Z^{\top}=L R^{\top} R L^{\top}=L S^{\top} L^{\top} L S L^{\top}$ (with $S$ as above). The non-zero eigenvalues of $L\left(S^{\top} L^{\top} L S L^{\top}\right)$ coincide with the non-zero eigenvalues of $\left(S^{\top} L^{\top} L S L^{\top}\right) L$. But $\operatorname{det}\left(S^{\top} L^{\top} L S L^{\top} L\right)=(\operatorname{det} S)^{2}\left(\operatorname{det} L^{\top} L\right)^{2}$ which is a nonzero rational square. Thus we have that the product of the non-zero eigenvalues of $Z Z^{\top}$ is a square, and Lemma 1 finishes the proof.

If $N$ is the incidence matrix of a self-dual design (that is, $N$ and $N^{\top}$ are isomorphic), then the left and right kernel of $N$ are obviously rationally equivalent and Lemma 2 gives:

Theorem 1 If $N$ is the incidence matrix of a self-dual design, then the product of the positive eigenvalues of $N N^{\top}$ is an integral square. 
For example if $N$ is the incidence matrix of a self-dual partial geometry with parameters $s(=t)$ and $\alpha$ (see [5]), the non-zero eigenvalues of $N N^{\top}$ are $(s+1)^{2}$ of multiplicity 1 , and $2 s+1-\alpha$ of multiplicity $s^{2}(s+1)^{2} / \alpha(2 s+1-\alpha)$. So if the latter multiplicity is odd, $2 s+1-\alpha$ is a square. In particular if $\alpha=1$, the partial geometry is a generalised quadrangle of order $s$ (denoted by $G Q(s)$ ) and we find:

Corollary 1 There exists no self-dual $G Q(s)$ if $s \equiv 2(\bmod 4)$ and $2 s$ is not a square.

For example no $G Q(6)$ is self-dual. Similarly, if $N$ is the incidence matrix of a generalised hexagon of order $s$ (denoted by $G H(s)$ ), the non-zero eigenvalues of $N N^{\top}$ are $(s+1)^{2}, s$ and $3 s$ of multiplicity $1, s(1+s)^{2}\left(1-s+s^{2}\right) / 2$ and $s(1+s)^{2}\left(1+s+s^{2}\right) / 6$, respectively (see for example [3] p. 203). Thus we find:

Corollary 2 There exists no self-dual $G H(s)$ if $s \equiv 2(\bmod 4)$.

Stronger condition are known if the incidence matrix of a $G Q(s)$ or $G H(s)$ is symmetric (see [9] p. 309). A symmetric incidence matrix clearly implies that the structure is selfdual, but the converse is not true in general (see [2] for an easy counterexample). Weaker conditions for the existence of self-dual generalised quadrangles were already found by Payne and Thas [6].

\section{Square divisible designs}

Another case when Lemma 1 can be applied is when the left and right kernel of $N$ are determined by the design requirements. Note that the left kernel of $N$ is the kernel of $N N^{\top}$, and similarly, the right kernel of $N$ is the kernel of $N^{\top} N$. So the lemma applies for square incidence matrices $N$ for which $N N^{\top}$ and $N^{\top} N$ are prescribed. For example, consider a 2- $(v, k, \lambda)$ design with a $v \times b$ incidence matrix where $b>v$. Extend the $v \times b$ incidence matrix with $b-v$ zero rows. For the $b \times b$ matrix $N$ thus obtained $N N^{\top}$ is known, and so is its left kernel. The right kernel of $N$ is in general not known, but there are some types of designs for which $N^{\top} N$ is prescribed. These include strongly resolvable designs and triangular designs. For these designs Bruck-Ryser-Chowla type conditions have been worked out; see $[4,7,8]$, so we will not do it again.

In this section we consider semi-regular square divisible designs. A divisible design (also called group-divisible design) with parameters $k, g, n, \lambda_{1}$ and $\lambda_{2}$, is an incidence structure, denoted by $G D\left(k, g, n, \lambda_{1}, \lambda_{2}\right)$, for which the points can be ordered such that the incidence matrix $N$ satisfies

$$
N N^{\top}=\lambda_{2} J_{v}+\left(\lambda_{1}-\lambda_{2}\right) K_{n, g}+\left(r-\lambda_{1}\right) I_{v} \text {, and } N^{\top} J_{v}=k J_{v},
$$

where $K_{n, g}$ is the block diagonal matrix $I_{n} \otimes J_{g}, v=n g$ is the number of points and $r=\left((n-1) g \lambda_{2}+(g-1) \lambda_{1}\right) /(k-1)$ is the replication number. The eigenvalues of $N N^{\top}$ are easily seen to be $k r, r-\lambda_{1}$, and $g\left(\lambda_{1}-\lambda_{2}\right)+r-\lambda_{1}$ with multiplicities $1, n(g-1)$ and $n-1$, respectively. Assume that $N$ is a square matrix. Then $r=k$, and the eigenvalues of 
$N N^{\top}$ become $k^{2}, k-\lambda_{1}$ and $k^{2}-g n \lambda_{2}$. If $N$ is non-singular, the divisible design is called regular, and necessary conditions for existence have been known for a long time, see [1], [7] p. 228, or [3] p. 23. If $N$ is singular, either $k=\lambda_{1}$ and $N=N^{\prime} \otimes J_{n}$, where $N^{\prime}$ is the incidence matrix of a square block design (then the divisible design is called singular), or $k^{2}=n g \lambda_{2}$ and the divisible design is called semi-regular.

Theorem 2 Let $D$ be a design with the property that both $D$ and its dual are a semi-regular $G D\left(k, g, n, \lambda_{1}, \lambda_{2}\right)$. Then

(i) if $g$ is even and $n$ is odd, $k-\lambda_{1}$ is an integral square,

(ii) if $g$ is even and $n \equiv 2(\bmod 4)$ then $k-\lambda_{1}$ is the sum of two integral squares,

(iii) if $g$ and $n$ are odd, the equation $\left(k-\lambda_{1}\right) X^{2}+(-1)^{(g-1) / 2} g Y^{2}=Z^{2}$ has an integral solution different from $X=Y=Z=0$.

Proof: Suppose $N$ is the incidence matrix of $D$. We may assume that $N N^{\top}=N^{\top} N$, which implies that $N^{\top}$ and $N$ have the same kernel, so by Lemma 2 the product of the non-zero eigenvalues of $N N^{\top}$ is a square, which proves $i$. Define $Z=\left(J_{n}-n I_{n}\right) \otimes J_{g}$. Then $\operatorname{rank} Z=n-1$, and $N N^{\top} Z=N^{\top} N Z=O$, so $Z$ satisfies the requirement for Lemma 1. Hence

$$
M M^{\top}=N N^{\top}+Z Z^{\top}=\left(\lambda_{2}-g n\right) J_{v}+\left(\lambda_{1}-\lambda_{2}+g n^{2}\right) K_{n, g}+\left(k-\lambda_{1}\right) I_{v} .
$$

has eigenvalues $k^{2}, \rho=k-\lambda_{1}$ and $\sigma=g^{2} n^{2}$ of multiplicity $1, n(g-1)$ and $n-1$ respectively. The Hasse-Minkowski invariant $C_{p}\left(M M^{\top}\right)$ with respect to the odd prime $p$ of a matrix $M M^{\top}$ of the above form is known, see for example [1].

$$
\begin{aligned}
C_{p}\left(M M^{\top}\right) & =(\rho,-1)_{p}^{n(g-1)(n+g-1) / 2}(\sigma,-1)_{p}^{n(n-1) / 2}(\sigma, g)_{p}^{n}(\rho, g)_{p}^{n}\left(\sigma, \lambda_{2}-g n\right)_{p} \\
& =(\rho,-1)_{p}^{n(g-1)(n+g-1) / 2}(\rho, g)_{p}^{n},
\end{aligned}
$$

where $(a, b)_{p}$ is the Hilbert norm residue symbol, defined by $(a, b)_{p}=1$ if for all $t$ the congruence $a X^{2}+b Y^{2} \equiv 1\left(\bmod p^{t}\right)$ has a rational solution, and $(a, b)_{p}=-1$ otherwise. Since $M$ is a non-singular rational matrix, $C_{p}\left(M M^{\top}\right)=C_{p}\left(I_{v}\right)=1$ for every odd prime $p$, and the conditions (ii) and (iii) follow.

For example there exists no $G D(18,4,9,6,9)$ for which the dual is also such a design. Note that in case $n=1, D$ is a square block design and the conditions are those of Bruck, Ryser and Chowla. The above theorem also has consequences for distance-regular graphs. Some putative distance-regular graphs imply the existence of square divisible designs (see [3] p. 22), and in case these divisible designs are semi-regular we obtain new conditions.

Corollary 3 Suppose there exists a distance-regular graph of diameter 4 with $2 g^{2} \mu$ vertices and intersection array $\{g \mu, g \mu-1,(g-1) \mu, 1 ; 1, \mu, g \mu-1, g \mu\}$. Then

(i) If $\mu$ is odd and $g \equiv 2(\bmod 4)$ then $g \mu$ is the sum of two integral squares.

(ii) If $\mu$ and $g$ are odd, then the equation $\mu X^{2}+(-1)^{(g-1) / 2} Y^{2}=g Z^{2}$ has an integral solution different from $X=Y=Z=0$. 
Proof: Such a distance-regular graph is the incidence graph of a $G D(g \mu, g, g \mu, 0, \mu)$ for which the dual is also such a design.

For example a distance-regular graph with intersection array $\{15,14,12,1 ; 1,3,14,15\}$ does not exist. Note that a distance-regular graph with intersection array $\{g \mu-1,(g-$ 1) $\mu, 1 ; 1, \mu, g \mu-1\}$ also gives rise to a semi-regular square divisible design; see [3], p. 24. But here we find no new restrictions.

\section{Acknowledgment}

I thank Edwin van Dam for many relevant conversations.

\section{References}

1. R.C. Bose and W.S. Connor, "Combinatorial properties of group divisible incomplete block designs," Ann. Math. Statist. 23 (1952) 367-383.

2. A.E. Brouwer, P.J. Cameron, W.H. Haemers, and D.A. Preece, "Self-dual, not self-polar," Discrete Math., to appear.

3. A.E. Brouwer, A.M. Cohen, and A. Neumaier, Distance-Regular Graphs, Springer, Heidelberg, 1989.

4. M.J. Coster and W.H. Haemers, "Quasi-symmetric designs related to the triangular graph," Designs, Codes and Cryptography 5 (1995), 27-42.

5. F. De Clerck and H. Van Maldeghem, "Some classes of rank 2 geometries," in Handbook of Incidence Geometry F. Buekenhout (ed.), Elsevier Science B.V., 1995, pp. 433-475.

6. S.E. Payne and J.A. Thas, "Generalized quadrangles with symmetry, Part I," Simon Stevin 49 (1975), 3-32.

7. D. Raghavarao, Constructions and Combinatorial Problems in Designs of Experiments, John Wiley \& Sons, Inc., 1971.

8. S.S. Shrikhande, D. Raghavarao, and S.K. Tharthare, "Non-existence of some unsymmetrical partially balanced incomplete block designs," Canad. J. Math. 15 (1963), 686-701.

9. H. Van Maldeghem, Generalized Polygons, Birkhäuser, 1991. 
